\title{
Carlos Alberto Seguín Escobedo (1907-1995): un peruano en la presidencia de la Sociedad Interamericana de Psicología
}

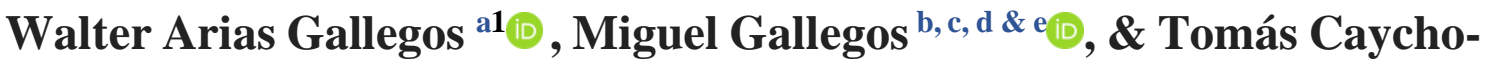 \\ Rodríguez ${ }^{\mathrm{f}}\left(\mathrm{C}^{2}\right.$
}

Universidad Católica San Pablo, Arequipa, Perúa ; Universidad Católica del Maule, Talca, Chile ${ }^{\mathrm{b}}$; Pontificia Universidade Católica de Minas Gerais, Belo Horizonte, Brasil c; Universidad Nacional de Rosario, Rosario, Argentina ${ }^{\mathrm{d}}$; Consejo Nacional de Investigaciones Científicas y Técnicas, Rosario, Argentina ${ }^{\mathrm{e}}$; Universidad Privada del Norte, Trujillo, Perú ${ }^{\mathrm{f}}$

\section{RESUMEN}

En este artículo revisamos la vida y obra de Carlos Alberto Seguín (1907-1995), uno de los psiquiatras más representativos del Perú, que contribuyó a difundir los saberes psicológicos y promovió el desarrollo de la medicina psicosomática en América Latina y planteó ideas originales en campos como la psiquiatría social y la psicoterapia, desde perspectivas psicoanalíticas y existencialistas. Presentamos primero un recorrido de sus primeros años y su formación como psiquiatra y psicoanalista, para luego dedicarnos al análisis de su obra y su participación en diversos eventos y organizaciones como la Sociedad Interamericana de Psicología. Además, se hace una revisión de su faceta docente, literaria y editorial.

Palabras Clave

Carlos Alberto Seguín; Sociedad Interamericana de Psicología; Historia de la Psicología; Perú; América Latina

\section{ABSTRACT}

In this article we present the life and work of Carlos Alberto Seguín (1907-1995), a renowned Peruvian psychiatrist, who disseminated the psychological knowledge and supported the development of psychosomatic medicine in Latin America, offering pioneering ideas in fields of social psychiatry and in psychotherapy based on psychoanalytic and existentialist perspectives. We provided an overview of his early years as a psychiatrist and psychoanalyst, followed by a discussion of his contributions in various events and professional organizations including the Interamerican Society of Psychology. Furthermore, we examined his academic career, his work publishing in peer reviewed journals, and later as an Editor.

Keyswords

Carlos Alberto Seguín; Interamerican Society of Psychology; History of psychology; Peru; Latin America.

\footnotetext{
1 Correspondence about this article should be addressed Walter Arias Gallegos: walterlizandro@ hotmail.com

2 Conflicts of Interest: The authors declare that the research was conducted in the absence of any commercial or financial relationships that could be construed as a potential conflict of interest.
} 
Carlos Alberto Seguín Escobedo (1907-1995): A Peruvian as President of the Interamerican Society of Psychology

\section{Introducción}

Carlos Alberto Seguín (1907-1995) fue uno de los psiquiatras más importantes de América Latina, que ha hecho una contribución significativa a la psicología peruana a través de su obra y diferentes actividades de gestión institucional (Alarcón, 2000, 2011). Por ello, Ardila (1971) ha señalado que sus libros constituyen hitos relevantes en la historia de la psicología latinoamericana. En ese sentido, Seguín se ha destacado en los campos de la psiquiatría social, la psiquiatría folklórica y la psicoterapia, favoreciendo la institucionalización de la psicología y la psiquiatría en el Perú.

Seguín ha trabajado temas propios de los saberes "psi" de forma original como la medicina psicosomática y el amor, proponiendo conceptos clave como el síndrome de psicosomático de desadaptación y el eros terapéutico. También ha publicado libros y artículos de investigación en diversas revistas de alcance nacional e internacional, ha editado revistas sobre saberes psi y ha ejercido el liderazgo de las instituciones más representativas de Latinoamérica como la Sociedad Interamericana de Psicología (SIP) y la Asociación Psiquiátrica de América Latina (APAL). Además, ha sido fundador de la Asociación Psiquiátrica Peruana y la Sociedad Peruana de Psicoterapia; y es considerado como una de las figuras más representativas del psicoanálisis en el Perú.

A pesar de todos sus logros y del indiscutible valor de sus aportes académicos y profesionales, poco se ha dicho de él en su ciudad de origen (Arias, 2016; Paredes, 2019), y no existen más que diversos textos breves, los cuales han repasado someramente sus contribuciones (Alarcón, 2011; Mariátegui, 1996; Perales, 2008). Entretanto, en el campo de la psicología, las obras más representativas de la historia de la psicología peruana (Alarcón, 2000; León, 1993) se han centrado sobre todo en Honorio Delgado (1892-1969) y Walter Blumenfeld (1882-1967). Es así que, con motivo del décimo séptimo aniversario de la SIP, se presenta un análisis de la vida y obra de Seguín, quien fuera presidente de la dicha sociedad entre 1965 y 1966, y presidente del X Congreso Interamericano de Psicología, llevado a cabo en el año 1966, en Perú (Hereford, \& Natalicio, 1967).

\section{Método}

Este estudio se enmarca dentro de las investigaciones teóricas-historiográficas caracterizadas por una revisión de estudios primarios sobre una temática de indagación 
específica (Ato, López-García, \& Benavente, 2013). Para este trabajo, se consultaron diversos archivos y bibliotecas con el fin de recuperar y analizar diferentes fuentes documentales primarias y secundarias de acuerdo a las investigaciones historiográficas (Klappenbach, 2014). Se relevaron y analizaron 34 documentos primarios escritos por Carlos Alberto Seguín desde 1938 a 1994, además de otros textos referidos a los aportes del autor. Aun cuando se reconoce que la producción de Seguín es mucho más extensa, en este trabajo solo se logró tener acceso a la documentación citada, la cual constituye el núcleo de las fuentes primarias consultadas. Las fuentes secundarias constituyen los aportes elaborados por otros autores sobre el periplo intelectual, científico, profesional y literario de Seguín.

Los materiales fueron analizados en tres etapas. En la primera, se recopilaron citas literales y compilación de resultados descriptivos de los aportes de Seguín, con el fin de ubicar sus áreas de trabajo más importantes y el contexto histórico y local en el que se desarrollaron. La segunda etapa se caracterizó por la revisión y análisis de diferentes estudios acerca de la obra y vida de Seguín, elaborados por diversos autores. En la tercera, se hizo una contrastación entre las dos fases anteriores, de modo que se logró realizar una discusión entre las fuentes primarias analizadas y las concepciones que otros autores han esbozado sobre la labor intelectual de Seguín. En su conjunto, este procedimiento facilitó el establecimiento y la organización del trabajo en cuatro ámbitos de desarrollo personal y profesional de Seguín: 1) su biografía personal; 2) sus aportes a la medicina psicosomática, psiquiatría social y psicoterapia; 3) su participación en organizaciones y eventos científicos; 4) y su vinculación con otros ámbitos de interés.

\section{Resultados}

\section{Raíces y formación de Carlos Alberto Seguín}

El propio Seguín indicó que su abuelo fue Manuel María Seguín, quien se casó con la dama arequipeña Sofía Delgado, hermana de Juan Ramón Delgado, padre del reconocido Honorio Delgado Espinoza; de ahí que Honorio Delgado fuera tío de Seguín. De la unión de sus abuelos, nacieron dos hijos, Sofía y Alberto Gonzalo, padre de Seguín, quien se casó con Emma Escobedo Arispe, proveniente de una de las familias más rancias de Arequipa, y tuvieron cuatro hijos: Carlos Alberto, Lila y Rosa (que eran gemelas) y Violeta. Seguín nació un jueves 8 de agosto de 1907 en la ciudad de Arequipa, ubicada al sur del Perú. Su niñez transcurrió entre las calles adornadas de sillar y los paisajes del 
balneario de Tingo. En este momento, se vivía una "República Aristocrática", caracterizada por el poder de los gobiernos civilistas que se fueron sucediendo desde 1899 hasta llegar a su apogeo en 1904 cuando José Pardo asume la presidencia hasta 1908, y es sucedido por Augusto B. Leguía entre 1908 y 1912 (Contreras, \& Cueto, 2018).

El padre de Seguín era periodista y padeció de cerca muchos avatares de la dictadura de Leguía, fundamentalmente durante la década de 1920, mientras se desarrollaba el segundo mandato presidencial entre 1919 y 1930 (Miro Quesada, 1957). El padre de Seguín fue director del diario "El Sol" del Cuzco y fundó el diario "El Heraldo" en Arequipa, el cual funcionaba en la casa familiar de los Seguín Escobedo. Su postura fue siempre democrática y descentralista, llegando a ser diputado durante el segundo gobierno de Leguía, y asestando desde sus funciones periodísticas y políticas, una feroz crítica contra el dictador. Por esta razón, Alberto Gonzalo Seguín fue exiliado en La Paz, Bolivia, donde pasó algunos años con su familia. También participó de una conspiración para derrocar a Leguía, que lo llevó a la prisión de Taquilla en el lago Titicaca, en Puno, donde eran enviados los opositores políticos del dictador (Silva, 1994).

Tras permanecer por 10 meses en Taquilla bajo condicionas inhumanas, Seguín padre fue deportado a Argentina, estableciéndose con su familia en Buenos Aires. Seguín hijo dejó el colegio cuando cursaba cuarto de secundaria para viajar a Buenos Aires en 1921 (Valdivia, 1991). Allí, el padre de Seguín consiguió un puesto como periodista que le permitió ganar un poco de dinero para solventar los gastos familiares, sin grandes comodidades. Tres años después, el 10 de octubre de 1924, muere Seguín padre y la familia retorna a Perú, pero Carlos Alberto Seguín se queda en Argentina para terminar sus estudios. Cursó el secundario en el Colegio Internacional de Olivos, un colegio que tenía fama de recibir a los alumnos que habían tenido fracasos escolares en los centros educativos de Buenos Aires, pero que provenían de familias pudientes. En ese colegio aprendió francés e italiano y se dedicó con ahínco a sus estudios, sobre todo, después de la muerte de su padre. En su adolescencia, y con el apoyo de un compañero, José Torres Nory, dirigió la revista Páginas del citado colegio, donde publicó algunos trabajos de corte literario y filosófico. También practicó deportes como natación, rugby y ajedrez, ganando una medalla en una competencia de natación y asumiendo el puesto de capitán en el Club de Rugby de Olivos.

Su padre era su confidente, su amigo y su consejero académico, quien le sugería lecturas y revisaba los textos que escribía por aquellos años. Por eso, la muerte de su padre le generó mucho dolor y un amargo sentimiento que le impulsó a seguir adelante. 
Manuel Lainez, director de "El Diario" donde trabajaba su padre, pagó los gastos del entierro y del viaje de retorno a Perú de toda la familia, pero Francisco Chelía, director del Colegio Internacional le sugirió quedarse en Argentina (Silva, 1994).

En 1926 ingresó a la Facultad de Medicina de la Universidad de Buenos Aires (UBA) (Valdivia, 1964). Para costear sus estudios trabajó como profesor de diversas materias, tales como ciencias naturales, francés, filosofía e historia en el Colegio Internacional. Siendo aún estudiante de medicina, publicó su primer libro en 1931, cuando tenía 24 años: Tratado de farmacología y terapéutica. Un año antes había publicado su primer artículo en la revista Día Médico con el título de "Curanderismo, psicoterapia y sugestión” (Alva, 1995) que hacía ver un preclaro interés por temas que le acompañarían a lo largo de su carrera.

En la UBA recibió clases de José Ingenieros (1877-1925) y de Bernardo Alberto Houssay (1887-1971), contagiándose del positivismo imperante. Durante su formación médica, estableció amistad con Florencio Escardó (1904-1992), médico que desarrolló innovaciones en el campo de la clínica pediátrica y la puericultura, llegando a ser Decano de la Facultad de Medicina y Vicerrector de la UBA. Escardó le dio un enfoque psicosocial a la pediatría argentina, al combinarla con la medicina psicosomática e introducir las escuelas para padres a finales de 1950, para lo cual se basó en las ideas de René Spitz, Edouard Claparède, Juan Rof Carballo y Helen Flanders Dumbar (Briolotti, 2017). Publicó, además una buena cantidad de artículos sobre enseñanza de la pediatría (Escardó, 1946) y medicina psicosomática infantil (Escardó, 1949); siendo una de sus obras más conocidas Anatomía de la familia (Escardó, 1962).

El 14 de abril de 1932, Seguín se graduó como médico-cirujano, y poco después se traslada a Formosa, donde a la edad de 26 años se casa con Dora Bellisomi, en 1936, a quien había conocido desde sus años de residencia en Buenos Aires. En esta región, ubicada al norte de Argentina, fue director del Hospital de Formosa y practicó la medicina general, la cirugía y la gineco-obstetricia (Alarcón, 2011), pero se dio cuenta que la clásica visión clínica era insuficiente para comprender a los enfermos que trataba. Por esta razón, regresa a Buenos Aires para especializarse en psiquiatría y toma contacto con la obra de Freud (Valdivia, 1991).

A este periodo corresponden sus primeras publicaciones sobre el psicoanálisis, que aparecieron en revistas argentinas, con una interpretación fiel a la ortodoxia freudiana (Seguín, 1938, 1940a). También publicó su segundo libro Freud un gran explorador del alma, en el que analiza la vida y obra de Freud, así como los fundamentos de la teoría 
psicoanalítica (Seguín, 1940b). Poco a poco, Seguín se fue aproximando a concepciones más ligadas a lo social, a través de la obra de Alfred Adler (1870-1937), que según Briolotti (2017), fueron insertadas en Argentina con la finalidad de dar orientaciones a los padres sobre la crianza de los hijos.

Estas son parte de las raíces del pensamiento social de Seguín, que le brindaron mayor apertura hacia otras orientaciones teóricas, sin desligarlas del psicoanálisis. De hecho, Seguín, mantuvo una postura holista que se nutre también de filósofos existencialistas, fenomenólogos y humanistas. Dicha perspectiva le llevó a criticar la práctica médica organicista y deshumanizada, y a desarrollarse como psiquiatra. En 1941, decidió regresar a Perú, y con la ayuda de Honorio Delgado pudo establecerse en Lima. Honorio Delgado lo incorpora al equipo editorial de la Revista de Neuro-psiquiatría, que había fundado en 1938 con Oscar Trelles (1904-1990) (Arias, 2015). Además, apoyó la publicación de sus trabajos y lo presentó ante las autoridades del Hospital Obrero, donde Guillermo Almenara, director del hospital le encarga atender las emergencias psiquiátricas, en el recién fundado nosocomio (Huarcaya-Victoria, 2016).

En 1942 obtuvo el grado de Doctor en Medicina con una tesis titulada “Contribución al estudio del hipnotismo", que sustentó en la Universidad Nacional Mayor de San Marcos (UNMSM), donde fue Jefe de Clínica de la cátedra de Psiquiatría (Perales, 2007). Ese mismo año, el Instituto Neuro-Psiquiátrico de Harttford (EE.UU.) ofreció una beca por concurso, la cual fue obtenida por Seguín. En Estados Unidos, se hizo amigo de Wladimir Liberson, director del Laboratorio de Electroencefalografía del Instituto Neuro-Psiquiátrico de Harttford. También fue alumno de Helen Flanders Dunbar (1902-1959), una de las más importantes exponentes de la medicina psicosomática en el mundo.

El término de medicina psicosomática fue acuñado por Johann Heinroth (17731843) en 1818, y luego rescatado por Felix Deutsch en 1922 (Ackerknecht, 1962); pero fue solo a través los avances en la neurofisiología del estrés (Selye, 1960) y la consolidación del psicoanálisis en Estados Unidos, que la medicina psicosomática alcanzó su mayor difusión. De este modo, en 1939 se fundó la Sociedad Psicosomática Americana que editó la revista Psychosomatic Medicine (Berrios, 2018). Flanders Dunbar fue una reconocida investigadora de la Universidad de Columbia que estudió la relación de las emociones y la salud física (enfermedades infecciosas, alergias, enfermedades respiratorias, gastrointestinales, cardiovasculares, genitourinarias, endocrino-metabólicas y degenerativas) dentro del marco de la medicina psicosomática (Flanders Dunbar, 1965). 
Entre 1944 y 1945, Seguín estudió con Flanders Dumbar y se incorporó como Research Associate in Psychiatry en la Universidad de Columbia, dictando clases y trabajando en el Presbiterian Hospital de Nueva York. Asistió a su vez, al grupo de estudio de Flanders Dunbar, con quien cultivó una gran amistad, y según narra el propio Seguín, le permitía usar su consultorio y le derivaba pacientes. También llevó cursos y seminarios en el Instituto Psicoanalítico de Nueva York, donde conoció a Nolan Lewis y Bertram Lewis, además de Roy Grinker en la Universidad de Columbia (Silva, 1994). Durante dos años se sometió a psicoanálisis didáctico con el vienés Richard Karper, de modo que pudo haber sido uno de los primeros peruanos en recibir una formación sistemática en psicoanálisis, más allá de que se reconoce a Honorio Delgado como una de las figuras más representativas del psicoanálisis en América Latina (Ardila, 1986; Gallegos, Falcone, Franco, \& Cervigni, 2019; León, 1992; Plotkin, \& Ruperthuz, 2017).

Si bien a Delgado se le reconoce haber introducido el psicoanálisis en el Perú (y también el tratamiento farmacológico), a partir de 1927 se distanció del psicoanálisis y desde 1940 fue un crítico mordaz. Puede decirse que más que psicoanalista, Delgado fue espiritualista (Arias, 2015). Por esta razón, los psicoanalistas peruanos, ven en Seguín a uno de los autores más representativos del psicoanálisis en el país, pues no solo recibió una formación sistemática en psicoanálisis, sino que además, fue siempre fiel a la doctrina de Freud. No ha quedado del todo esclarecido por qué Honorio Delgado se tornó beligerante con el psicoanálisis, aun cuando nunca dejó de escribir acerca del psicoanálisis y la propia persona de Freud (Gallegos, Falcone, Franco, \& Cervigni, 2019; Gallegos \& Franco, 2020).

En el análisis cronológico sobre la obra psicoanalítica de Delgado, Seguín señala que su tío se revela en contra de las ideas que Freud escribió sobre religión, la vida instintiva, el énfasis en lo sexual, y posiblemente por el origen judío de Freud. De esta manera, y en base a los cambios que se aprecian en los textos que escribe Delgado, Seguín piensa que éste renuncia a su adhesión al psicoanálisis para congraciarse con la sociedad y la Iglesia, pues por aquellos años cultiva amistad con José de la Riva Agüero y Víctor Andrés Belaunde (Seguín, 1982), dos de las figuras más destacadas del intelectualismo católico en el Perú; y colabora publicando artículos en la revista Mercurio Peruano, que por aquellos años editaba Belaunde.

La relación entre Delgado y Seguín fue al principio muy cordial y cercana, pues varias de las publicaciones de Delgado, y sobre todo sus libros, eran obsequiados y dedicados a su sobrino (Huarcaya-Victoria, 2016); pero luego se tornó un poco distante, 
aunque Seguín valoraba más las virtudes que los defectos de su tío, por quien nunca dejó de sentir admiración (Silva, 1979). Asimismo, existen varias similitudes entre ambos, además de ser familiares, Delgado como Seguín, fueron psiquiatras con un notable interés por la psicología. Ambos publicaron diversas obras sobre psicoanálisis, pero Delgado la combinó con la higiene mental y la eugenesia, mientras que Seguín se orientó por la medicina psicosomática y la psiquiatría social. Desde luego, tuvieron sus diferencias, algunas sutiles y otras más determinantes. En el primer caso, si bien ambos reconocieron el valor de la filosofía, Delgado se orientó por la fenomenología y Seguín por el existencialismo. En el segundo caso, aun cuando ambos fueron católicos, Delgado fue más autoritario, elitista y puritano; mientras Seguín fue más humanitario, con una elevada sensibilidad social y con un vivo interés académico por lo "erótico".

\section{Medicina psicosomática, psiquiatría social y psicoterapia}

Cuando Seguín estuvo en Nueva York se le ofreció la posibilidad de quedarse en Estados Unidos y hacer su carrera académica en la Universidad de Columbia, pero la añoranza de volver con su esposa e hijo, le hicieron declinar ante dicha propuesta. En 1945, Seguín retorna a Perú y se instala con su familia en Lima, pero esta vez, con una fuerte determinación para reformar la psiquiatría peruana (Alarcón, 2011), que por su orientación organicista tendía a cosificar al ser humano, alejándose de su verdadera finalidad. Es aquí que comienza un periodo de gran productividad para Seguín, que le permite ganar reconocimiento y posicionarse como una de las figuras más célebres de la psiquiatría peruana (Valdivia, 1964).

Una vez instalado en Perú, decide poner en práctica todo lo aprendido y promover fuertemente la medicina psicosomática, formando en el Hospital Obrero un grupo de trabajo e investigación, conocido simplemente como el Grupo Obrero y otras como Escuela de Psiquiatras de la Caja Nacional del Seguro Social Obrero, que se constituyó en la primera escuela de psiquiatras del país, en la que se formaron varios psiquiatras de Perú y el extranjero (Huarcaya-Victoria, 2016). En este servicio de psiquiatría, que según Alarcón (2011) sería el primero en fundarse dentro de un hospital general en América Latina, se condujeron seminarios, discusiones de casos y análisis de la literatura científica sobre medicina psicosomática, que atrajeron a varios profesionales de la medicina, la psicología y disciplinas afines. 
El Grupo Obrero realizó investigación clínica y terapéutica, y se convirtió en un foco de actividad de la medicina psicosomática en el Perú; pero esto no fue tan fácil, dado que los médicos del hospital se mostraron renuentes a participar de las reuniones clínicas en las que se explicaba el enfoque psicosomático y un abordaje más integral del tratamiento médico de los pacientes (Silva, 1994). En 1946, Seguín se hizo cargo de la cátedra de "Psiquiatría" de UNMSM bajo la jefatura de Delgado y también dictó la cátedra de "Semiología psicosomática" (Alarcón, 2011), introduciendo un modelo de historia clínica psicosomática (Seguín, s/f). Pero por entonces, se distanció un poco de Delgado, pues mientras Seguín se apasionaba cada vez más con el psicoanálisis y su vertiente psicosomática, Delgado criticaba a Freud y descalificaba sus métodos (Seguín, 1982).

\section{Figura 1}

Carlos Alberto Seguín (1907-1995)

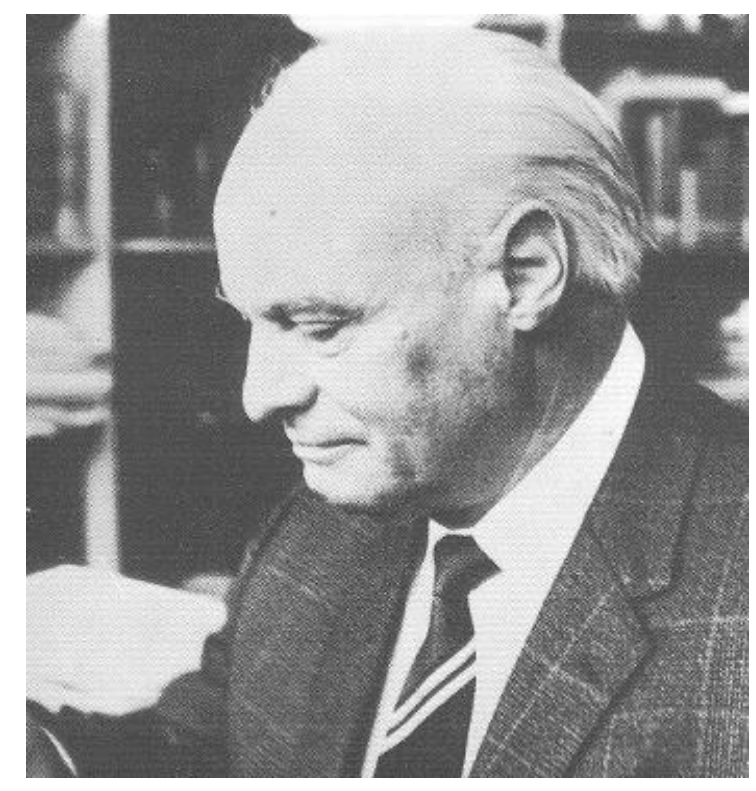

Parte de la investigación del Grupo Obrero surgía de las tesis de los alumnos de Seguín, que tocaban temas como la personalidad de los pacientes con apendicitis crónica, en la que se predijo que las personas con una personalidad neurótica no mejorarían luego de la intervención quirúrgica, sino que tendrían nuevas patologías. Con el éxito de estos estudios, fue ganando credibilidad la medicina psicosomática $\mathrm{y}$, poco a poco, la concurrencia a los eventos organizados por Seguín fue creciendo, hasta ser consultado por dermatólogos, gastroenterólogos y diversos especialistas sobre los componentes psicológicos de las dolencias de sus pacientes. En 1946 funda el Centro de Estudios Psicosomáticos, que congregaba a varios especialistas de América Latina, y en 1947 
publica Introducción a la Medicina Psicosomática, obra en la que expone los fundamentos del enfoque psicosomático y la atención integral del paciente (Seguín, 1947), que le mereció el Premio Nacional Hipólito Unanue, siendo traducida al inglés y al francés (Romero, 2007). En este texto Seguín es enfático en señalar que:

Debemos insistir desde ahora en la noción de que las homeostasis fisiológica y psíquica no son en esencia separables sino que, por el contrario, se nutren mutuamente y reaccionan de un modo paralelo, lo que nos lleva al concepto de unidad psicosomática. (Seguín, 1947, p. 36)

En 1950 publica la revista Estudios Psicosomáticos, pero tuvo que ser suspendida por las dificultades que implicaba su distribución internacional (Silva, 1979). Asimismo, en 1949 Seguín fue incorporado como miembro del comité editorial de la revista Psychosomatic Medicine y en 1955 fue elegido como Segundo Vicepresidente de la American Academy of Psychosomatic Medicine. De hecho, Seguín publicó varios trabajos de medicina psicosomática en revistas nacionales e internacionales, tanto sobre la formación de los estudiantes de medicina en esta corriente (Seguín, 1946b), como sobre el abordaje clínico integral desde una perspectiva psicosomática (Seguín, 1952), y bautizando como "complejo de Antíoco" a la versión psicosomática del complejo de Edipo (Seguín, 1961a). Muchos de estos trabajos se publicaron en la Revista del Hospital Obrero, los Anales del Servicio de Psiquiatría del Hospital Obrero y en la revista Psychosomatic Medicine (Seguín, 1946a, 1949, 1956), donde dio a conocer el "síndrome psicosomático de desadaptación”, uno de sus aportes más originales a la psicología peruana (Alarcón, 2000).

Este síndrome era producto de la difícil situación que le tocaba vivir a los migrantes de la sierra peruana, que buscando mejores oportunidades laborales, se instalaban en la ciudad de Lima, siendo víctimas de humillaciones y abusos por su bajo nivel educativo y sus costumbres, que terminaban por sumirlos en la ansiedad, depresión, una variedad de somatizaciones y trastornos psicosomáticos (Seguín, 1990b). Seguín encontró que los migrantes peruanos padecían alteraciones circulatorias, digestivas, respiratorias, musculares y nerviosas; además de cefaleas, depresión y angustia. En palabras de Seguín: 
Obligados a variar bruscamente sus condiciones de vida, sufren intensamente por el cambio de clima, de alimentación, de costumbres y por su posición de inferioridad frente al costeño que se burla de ellos y los hostiliza; por la dureza del trabajo, por la dificultad del lenguaje y por el traumatismo fisiológico de adaptación que hemos visto estudiado por Monge y su escuela $\mathrm{y}$, en gran porcentaje, ven sobrepasada su capacidad psicosomática de adaptación y se enferman. (Seguín, 1990b, p. 139)

\section{Y agrega:}

En realidad, el síndrome de desadaptación es solamente un caso particular y exagerado del común fenómeno de la nostalgia, la "morriña" o el "saudade". La importancia de su descripción está en que constituye una reacción frecuentísima en Lima y en que su verdadero significado no es comprendido porque se enfoca la atención en el examen de los órganos y no se piensa en el individuo, lo que ocasiona una completa desorientación diagnóstica y terapéutica. (Seguín, 1990b, p. 141)

Este síndrome era más frecuente en personas indígenas o mestizas, de entre 15 y 25 años, que habían migrado de sus comunidades, donde se dedicaban a las labores agrícolas en la sierra del Perú; y cuyo pronóstico dependía de la personalidad del paciente, de la gravedad del síndrome fisiológico, de la edad cronológica y el tiempo de evolución de la enfermedad. Todos estos aportes en el campo académico y profesional, generaron que la medicina psicosomática tuviera una buena acogida en Perú, donde el grupo de médicos y psicólogos interesados por dicho enfoque fue creciendo. De hecho, el curso de "Semiología psicosomática" que dictaba Seguín se reabrió como curso libre en 1955, tras ser suprimido en 1951, muy a pesar de los deseos de los estudiantes de tercer año de la carrera de Medicina de la UNMSM. Para esa fecha, las redes académicas de Seguín en América Latina abarcaban a varios especialistas interesados en medicina psicosomática como Luis Dragunski, Roberto Villalón, César Ottalagano, Enrique Luque, Rafael Sorol, Héctor Warnes de Argentina, Hernán Saavedra de Colombia, María Helena de Souza de Brasil, Julio Gutiérrez de Nicaragua, Mario Mendoza de Honduras y José Patxot de República Dominicana, entre muchos otros (Valdivia, 1964). 
Los temas desarrollados por Seguín en medicina psicosomática y el peso de los factores culturales en el síndrome psicosomático de desadaptación, le acercaron cada vez más a la psiquiatría social, tema que en América Latina cobró fuerza en la década de 1960 como consecuencia de la inserción de los estudios antropológicos y sociológicos que impactaron en medicina, así como de los enfoques basados en la epistemología crítica (Ferreira, 2010). De este modo, los modelos basados en la higiene mental dieron paso a los modelos de salud mental con fuerte acento en lo social (Dagfal, 2015). Seguín fue muy receptivo a estas ideas, primero porque desde su etapa formativa en Argentina tomó conciencia del carácter intersubjetivo de la profesión médica, ya que "la medicina no se trata de enfermedades sino de enfermos" (Seguín, 1957, p. 47); de ahí que desarrolló una sólida vocación genuinamente humanista (Mariátegui, 1996), que le permitió acercarse al conocimiento filosófico como base de las ideas en el quehacer psiquiátrico (Berrios, 2011).

En esa dirección, Seguín publicó en 1960 el libro Existencialismo y psiquiatría, en el que se dedica al análisis de las relaciones entre la filosofía y la psiquiatría desde un enfoque interpersonal (Seguín, 1960), tomando como base las ideas de Martín Buber (1974/2017), Martín Heidegger (1987/2003), Jean-Paul Sartre (1943/2008), etc. En estos autores, entre otros, encontró una aproximación afín a su concepción integral del "ser" humano. De acuerdo a Silva (1994), en esa obra, Seguín también introduce en el Perú la teoría del análisis transaccional, ya que además, desde 1974 inicia en el Hospital Obrero seminarios de discusión de la teoría de Eric Berne (Valdivia, 1991). Esta visión integral le plantea la necesidad de focalizarse en el tejido interpersonal de la relación médicopaciente, que sería determinante de su trabajo terapéutico y sus trabajos en psiquiatría social, que como ya hemos señalado, también estuvieron impulsados por sus experiencias con los pacientes migrantes que atendía en el Servicio de Psiquiatría del Hospital Obrero.

La psiquiatría social fue objeto de estudio de varios psiquiatras en el Perú, y es considerada por Alarcón (2000) como uno de los aportes más destacables de la psiquiatría al desarrollo de la psicología peruana, ya que constituyó un puente entre ambas disciplinas por el abordaje interdisciplinario que supone, y por el aporte docente que desembocó en el desarrollo de una psicología social orientada al estudio de las poblaciones que vivían en la marginalidad. Esto derivó en la publicación del libro Psiquiatría y sociedad, que compila varios trabajos de psiquiatras que trabajaban en dicho campo (Seguín, 1962). Sin dudas, este fue el periodo en el que la psiquiatría social se desarrolla en el Perú. Para esos años, otros autores publicaron Estudios de psiquiatría social en el Perú (Caravedo, 
Rotondo, \& Mariátegui, 1963) en el que psiquiatras y trabajadores sociales colaboraron para investigar, a través de la aplicación de diversas pruebas proyectivas y psicológicas, la salud mental de los habitantes de zonas urbano-marginales de Lima.

Humberto Rotondo (1915-1985), otro psiquiatra ilustre que trabajó la psiquiatría social en el Perú y coautor del texto aludido, desarrolló también fuertemente el campo de la psiquiatría social en el país (Rotondo, 1959, 1967; Rotondo, Mariátegui, Bambarén, García, \& Aliaga, 1962) y colaboró con Seguín en algunos trabajos (Caravedo, Seguín, \& Rotondo, 1963; Caravedo, Rotondo, Seguín, Perales, \& Blanco, 1971), dado que tenía afinidad por la medicina psicosomática y el psicoanálisis (Rotondo, 1949). Parte de su trabajo tuvo que ver con la reforma psiquiátrica enfocada en las comunidades terapéuticas, que suponen la vinculación de los pacientes con la sociedad a través de la laborterapia, la musicoterapia, la arteterapia, y otras modalidades de tratamiento inspiradas en la obra de Maxwell Jones (1907-1990), que propugnó una psiquiatría menos restrictiva y más abierta a la comunidad, como resultado de la crítica de Thomas Zsasz (1970/2006) y Michel Foucault (1984, 1963/2013). Seguín señala, por ejemplo, que en la comunidad terapéutica, los individuos no son el foco sino el grupo, enfatizando el enfoque social del tratamiento (Seguín, \& Buckley, 1971).

En ese sentido, un campo en el que Seguín también contribuyó a fortalecer en el Perú, es el de la psicoterapia (Peña, 2010), sobre la cual ha escrito varios de sus trabajos (Seguín, 1953/2007, 1963, 1965, 1970, 1990a). En 1953 publica Bases de la psicoterapia, en el que explica diversos modelos psicoterapéuticos (Seguín, 1953/2007) y en 1963 publica Amor y psicoterapia, en el que introduce el concepto de "Eros terapéutico", que Seguín diferencia del amor amical, pastoral, parental, fraternal, erótico y pedagógico; definiéndolo como:

...un amor por el paciente o, mejor aún, por la persona del paciente. Lo que quiero decir es que no se trata de un amor "humanitario" que el médico debe sentir por el enfermo, sino de un movimiento auténtico hacia el individuo particular que se halla ante él, que es éste y no otro y que no es un enfermo sino un hombre. (Seguín, 1963, p. 106-107)

Para Seguín, la psicoterapia es una forma de amor basado en los valores personales que busca la autorrealización del paciente. Así pues, Seguín se alinea con las ideas de Carl Rogers (1997), Viktor Frankl (1987/1997) y diversos pensadores existencialistas, 
que proponen una terapia no directiva, en la que la experiencia terapéutica es construida tanto por el paciente como por el terapeuta. Este carácter dual del abordaje psicoterapéutico es básico de toda relación humana, y Seguín lo complementa con la escucha activa y el análisis del lenguaje centrado en la comunicación interpersonal, temas que retoma en su libro El quinto oído (Seguín, 1990a). Romero (2007) ha señalado que Seguín integró el psicoanálisis con la filosofía existencial derivando en una propuesta terapéutica sui generis.

Aunque Seguín define la psicoterapia como una disciplina médica, incorpora diversos conceptos psicológicos (Alarcón, 1975). En ese sentido, debe señalarse que la visión psiquiátrica de Seguín, dada su orientación teórica basada en el psicoanálisis y existencialismo, y su actividad práctica fundada en la medicina psicosomática y la psicoterapia, fue siempre abierta a la psicología, y no la consideró solo como una disciplina accesoria de la psiquiatría, como era menester de parte de los médicos y psiquiatras de mitad del siglo XX, sino que le dio su lugar como disciplina independiente que hacía una sólida contribución a las ciencias de la salud, y la incluyó como parte vital de la formación médica (Seguín, 1964b).

En 1962 cuando fue nombrado jefe del Departamento de Psicología de la Facultad de Medicina de la UNMSM, renombra dicha instancia como Departamento de Ciencias Psicológicas y Neurológicas con la finalidad de dar mayor difusión a la medicina psicosomática (Perales, 2008), revalorando las relaciones entre la medicina y la psicología. Seguín asume esta jefatura y la titularidad de la cátedra de "Psiquiatría" en la UNMSM, luego de que Delgado y alrededor de 40 docentes de Medicina, renuncian a sus cargos en dicha casa de estudios como protesta por las reformas universitarias que incluían representantes estudiantiles en los órganos de gobierno de las universidades peruanas en 1960 (Porturas, 1999).

$\mathrm{Al}$ asumir estas funciones de gestión y docencia, Seguín creó grupos formativos entre estudiantes y profesores e instauró su proyecto de medicina psicosomática. En ese marco, publica en 1964 el libro La preparación psicológica del estudiante de medicina en el que expone su proyecto sobre la base de las experiencias formativas en otros países donde la psicología es parte esencial del currículo en la formación médica, además de presentar la reforma curricular, los métodos y las horas de clase con una mayor presencia de cursos relativos a la salud mental desde el primer hasta el quinto año (Seguín, 1964a). Funda además en ese año, la Revista de Ciencias Psicológicas y Neurológicas como órgano de dicho departamento, que tuvo una importante difusión en el país y el extranjero 
(Alarcón, 2000). Pero todas estas actividades se frustraron debido a que un núcleo de profesores y alumnos socavaron el proyecto, de modo que Seguín da un paso al costado en 1969 señalando en su carta de renuncia que "no desea enseñar a alumnos que no quieren aprender" (Perales, 2007, p. 16).

Con el tiempo, Seguín se comprometió cada vez más con los estudios de psiquiatría social, con una orientación cercana a la obra de Erich Fromm (1900-1980) y Karen Horney (1885-1952), pero volcándose hacia la psiquiatría folklórica. Esta temática había sido abordada sistemáticamente por Hermilio Valdizán (1885-1929) entre los años 1913 y 1925 (Valdizán, 1913, 1918a, 1818b, 1925) dentro del campo que denominó etnopsiquiatría, que se aboca a la evaluación psicológica y psiquiátrica de los indígenas peruanos, concluyendo —en base a las teorías de la degeneración- que los indígenas peruanos tienen disminuida su capacidad intelectual debido al consumo de la hoja de coca y de diversas bebidas alcohólicas (Valdizán, 1990). Los estudios de Valdizán se ubican en el proyecto que llevó a cabo con el fin de desarrollar una psiquiatría peruana (Mariátegui, 1981), y que sirvió para legitimar diversos prejuicios raciales de la época (Orbegoso, 2016).

Varios psiquiatras peruanos también trabajaron temáticas similares, como Carlos Gutiérrez Noriega, quien investigó los rasgos psicopatológicos en indígenas desde una perspectiva biotipológica (Gutiérrez Noriega, 1936, 1937, 1939), Federico Sal y Rosas, quien investigó la paleopsiquiatría y el pensamiento mágico de los antiguos pobladores del Perú (Sal y Rosas, 1965, 1967) y Mario Chiappe, quien investigó la práctica del curanderismo en diversas regiones del país (Chiappe, 1969, 1972 1989), entre muchos otros. En el caso de Seguín, su interés se centró en la psiquiatría folklórica a través de diversas publicaciones (Seguín, 1969, 1971, 1972, 1979, 1988), siendo las más importantes Psiquiatría folklórica (Seguín, 1979) y Medicinas tradicionales y medicina folklórica (Seguín, 1988) en las que define a la psiquiatría folklórica como el estudio “de las ideas, las creencias y las prácticas que se refieren a los cuadros psiquiátricos y su tratamiento, mantenidas por tradición popular; aparte y en contra de los aceptado por la cultura dominante en el medio en que se presentan" (Seguín, 1979, p. 21). 


\section{Figura 2}

Portada del libro Psiquiatría folklórica

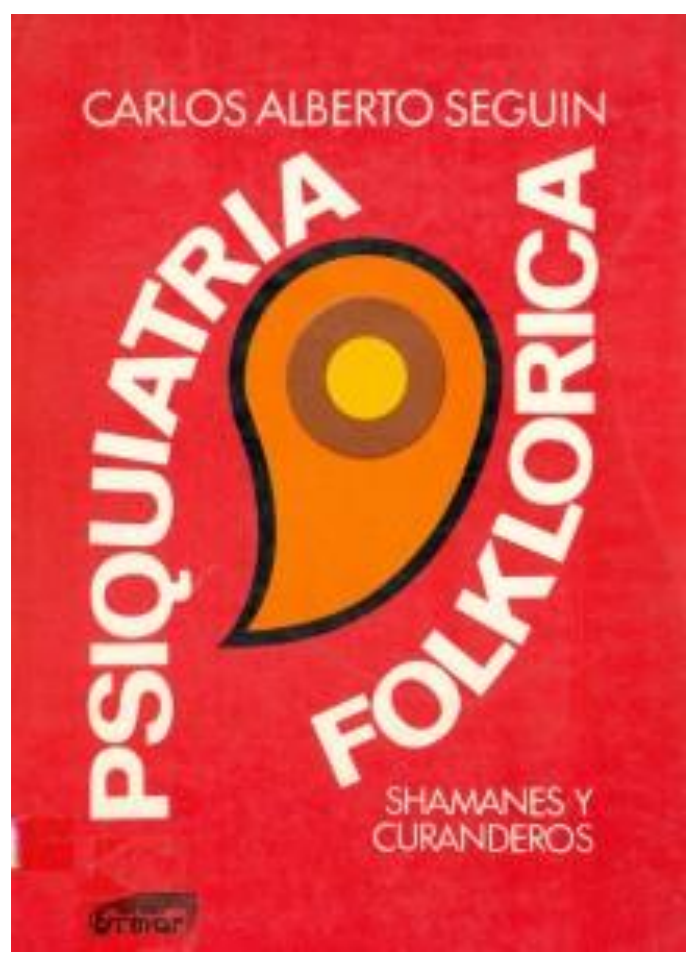

Nota: Tomado de Psiquiatría folklórica (p.1), C. A. Seguín, 1979, Ediciones Esrmar.

Además, distinguió entre la medicina académica, la etno-medicina, la medicina tradicional integrada, la medicina popular y la medicina folklórica, campo dentro del cual ubicó a la psiquiatría folklórica en relación con la historia de la medicina folklórica, la antropología cultural, la sociología folklórica, la nosografía folklórica, el psicoanálisis, la patogénesis folklórica, la terapéutica folklórica y la medicina folklórica transcultural y comparativa (Seguín, 1988).

Seguín estudió los fenómenos del susto y el daño, entre otros, otorgándoles el grado de categoría clínica (Romero, 2007), pues para él tiene gran relevancia cultural y antropológica el comprender las diversas formas de la medicina folklórica, y estas no deben ser despojadas de su valor psicosocial en las diversas comunidades del mundo, sean países industrializados o en vías de desarrollo (Seguín, 1972). En palabras de Seguín:

...la medicina folklórica debe ser considerada, no solamente como una forma anticientífica de tratar las enfermedades, sino como mucho más: deriva de conceptos del hombre y de la vida diferentes de aquellos que rigen en nuestro ambiente occidentalizado y no debe verse desde el punto de vista de si llena o no 
las condiciones del conocimiento científico, sino poner el acento, sobre todo, en su significado, en su función y su valor humano. (Seguín, 1988, p. 86-87)

Seguín señala que aunque la psiquiatría folklórica merece ser un tema legítimo de estudio científico de parte de la medicina académica, y la práctica que ejercen brujos, chamanes y curanderos, también tiene que ser vista con respeto; debe distinguirse de la charlatanería (Seguín, 1979). Asimismo, Seguín desarrolló una propuesta de investigación para la psiquiatría folklórica, que abarca el estudio de los curanderos, el ambiente donde se realiza el tratamiento, las bases diagnósticas, el seguimiento de los pacientes, las relaciones locales y extralocales con otros miembros de la comunidad, el estudio de las plantas usadas y sus efectos farmacológicos (Seguín, 1988).

\section{Participación en organizaciones y eventos científicos}

En 1950, Seguín participó del Primer Congreso Mundial de Psiquiatría en París, con una ponencia dedicada a la medicina psicosomática. Este congreso fue organizado por Henry Ey, y quedó establecida una comisión de los congresos mundiales de psiquiatría a su cargo. Durante este evento se fundó la Asociación Psiquiátrica Mundial, de la cual Seguín participó como directivo. Aquel primer congreso dejó una huella imborrable en Seguín, dado que conoció personalmente a Ana Freud, Franz Alexander, Eugen Bleuler, Carl Jung y Henry Ey, entre otros; pero además, porque su viaje se ensombreció con la muerte de un amigo y muy destacado psiquiatra peruano, con quien Seguín viajó al congreso de París. Nos referimos a Carlos Gutiérrez Noriega (1906-1950), un psiquiatra que trabajó la epistemología de las ciencias y la psiquiatría experimental, además de la etno-psiquiatría (Mariátegui, 1985). Resulta que trasladándose a Pisa (Italia), mientras conversaban sobre diversos temas, Seguín y Gutiérrez Noriega se detuvieron para admirar el paisaje, cuando un vehículo pesado los impactó, ocasionando la muerte instantánea de Gutiérrez Noriega y dejando grave a Seguín, con varias costillas rotas, un tobillo desplazado, un brazo quebrado y una reacción anafiláctica al suero antitetánico que le administraron en el hospital (Silva, 1994).

Seguín participó también del grupo constituido por Raúl González Enríquez y Guillermo Dávila (ambos de México) y José Ángel Bustamante (de Cuba), que en el marco del IV Congreso Internacional de Salud Mental llevado a cabo en México en 1951, formó el Grupo Latinoamericano de Estudios Transculturales (GLADET) y la 
Asociación Psiquiátrica de América Latina (APAL), la cual demoró su consolidación debido al prematuro fallecimiento de González Enríquez, el principal promotor de dicha iniciativa (Campos-Farfán, 2017). No obstante, gracias a sus miembros fundadores, la APAL se fortaleció paulatinamente y logró organizar diversos congresos de psiquiatría en los diferentes países de la región desde 1951. El congreso de 1964, celebrado en el Perú, estuvo presidido de Seguín (Seguín, \& Ríos, 1964).

Por otro lado, también en 1951 se crea la Sociedad Interamericana de Psicología (SIP) en México, con la participación de un grupo de psicólogos y psiquiatras que estuvieron presentes en el citado IV Congreso Internacional de Salud Mental (Ardila, 1986, Gallegos, 2012a). En 1953 se organiza el I Congreso Interamericano Psicología en Santo Domingo (República Dominicana) con Andrés Avelino García como presidente del comité organizador. En este evento participaron varios especialistas de Perú, entre quienes podemos mencionar a Walter Blumenfeld, Honorio Delgado, Carlos Alberto Seguín, Leopoldo Chiappo y Emilio Majluf; pero algunos de ellos no pudieron estar presentes físicamente. Tal fue el caso de Seguín, quien presentó una disertación titulada "Bases de la psicoterapia”, aunque su ponencia fue leída, ya que Seguín no pudo asistir (Gallegos, 2012b). En esta ponencia Seguín se centra en la comunicación y las relaciones terapeuta-paciente, analizando diversos modelos teóricos y enfatizando los aspectos intersubjetivos y emocionales que intervienen en dicho proceso (Seguín, 1955).

Entre 1965 y 1966 Seguín asumió la presidencia de la SIP y presidió X Congreso Interamericano de Psicología, que tuvo lugar en Lima, entre el 3 y 7 de abril de 1966 (Arias, 2014). En este evento se presentaron 93 ponencias y concurrieron 466 asistentes, participando como miembros del comité organizador, destacados exponentes de la psicología y la psiquiatría peruanas como Segisfredo Luza, Baltazar Caravedo Carranza, Max Hernández, Reynaldo Alarcón, Arnaldo Cano, Raúl González Moreyra, Federico León, Gustavo Saco y Carlos Franco, entre otros (Hereford \& Natalicio, 1967). 


\section{Figura 3}

Carlos Alberto Seguín en el X Congreso Interamericano de Psicología (Lima, 1966).

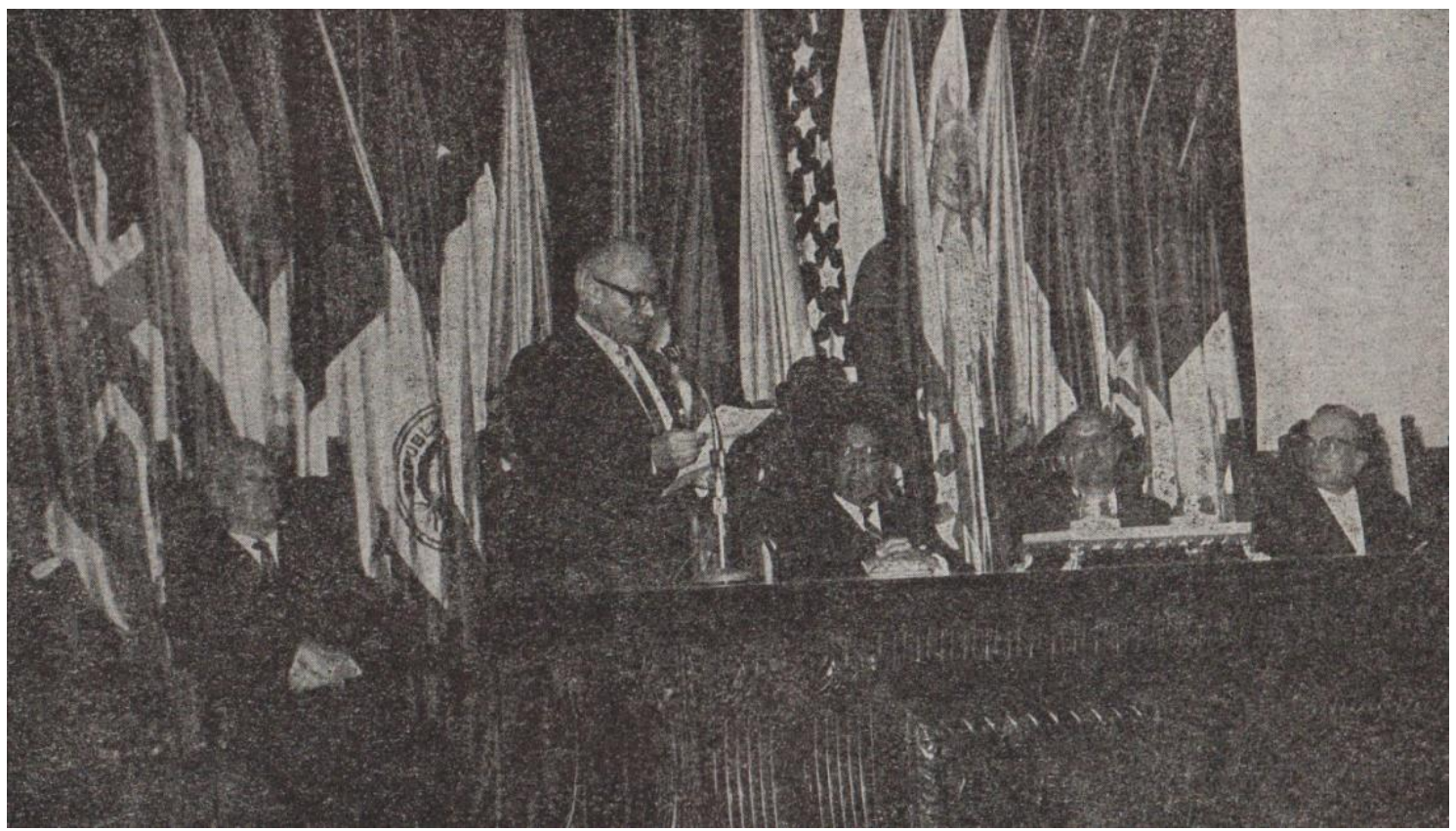

Nota: Tomado de Aportaciones de la psicología ala investigación transcultural.

Memorias del Décimo Congreso de la Sociedad Interamericana de Psicología (p. 45)

C. F. Hereford \& L. Natalicio (Comps.), 1967, Trillas.

Seguín ha tenido una importante participación en instituciones vinculadas a la

salud mental y en numerosos eventos académicos alrededor del mundo. En 1954 se funda la Asociación Psiquiátrica Peruana por obra de Seguín, Francisco Alarco Larraburre (1915-1990), Baltazar Caravedo Carranza (1915-1990) y Arnaldo Cano Jáuregui (19191987), siendo su primer presidente Federico Sal y Rosas (1900-1974) (Valdivia, 1991). En 1957 esta asociación publicó la Revista Psiquiátrica Peruana y en 1969 organizó el Primer Congreso Nacional de Psiquiatría, en Lima (Valdivia, \& Péndola, 1969), en el que Seguín participó con una ponencia sobre psiquiatría folklórica (Seguín, 1969). Asimismo colaboró, conjuntamente con otros psiquiatras, para fundar la Sociedad Peruana de Psicoterapia en 1979, la cual editó la Revista Peruana de Psicoterapia, desde 1985 (Cabrejos, 1995).

Fue presidente de la Asociación Psiquiátrica Peruana y del Centro de Estudios Psicosomáticos, fue coordinador del Instituto Peruano de Antropología y Psiquiatría, secretario general de la Sociedad de Neuro-Psiquiatría y Medicina Legal, vice-presidente del Cuerpo Médico del Hospital Obrero, miembro de la comisión de la Asociación Internacional de Psicología Médica y de la comisión organizadora de la Asociación Internacional de Psicoterapia de Grupo, miembro de GLADET, miembro de la Federación Mundial de Salud Mental, fundador de la Academy on Religion and Mental 
Health, Vice-presidente de la American Academy of Psychosomatic Medicine, presidente de la SIP, miembro de la Sociedad Peruana de Historia de la Medicina, miembro de la American Psychiatric Association, de la American Psychosomatic Society y de la Association for Advancement of Psychotherapy, entre otras sociedades psiquiátricas de los países de América Latina (Valdivia, 1991).

Seguín también fue un activo participante de congresos nacionales, regionales e internacionales a mediados del siglo XX. Fue ponente del Primer Congreso Mundial de Psiquiatría en 1950, presidiendo la comisión de Psicoanálisis del comité peruano, y al año siguiente participó del Segundo Congreso Mundial de Psiquiatría en Zurich. También participó del IV Congreso Internacional de Salud Mental, celebrado en México en 1951, del Congreso Mundial de la Federación Mundial para la Salud Mental en Viena en 1953 y su homólogo en 1954 que tuvo lugar en Toronto. Ese mismo año participa del Congreso Latinoamericano de Psiquiatría celebrado en Chile, y en 1955 del Quinto Congreso de la Conferencia Médica Panamericana en Bogotá. En 1956 participó del Segundo Congreso Ibero-Americano Médico Psicológico que tuvo lugar en Buenos Aires, y en 1958 del Congreso Internacional de Psicoterapia celebrado en Barcelona. En 1960 participa de la Conferencia Médica Panamericana en México, en 1961 participa del Quinto Congreso Mundial de Psiquiatría celebrado en Viena, y el mismo año participa del Sexto Congreso de la Federación Mundial de Salud Mental en París (Valdivia, 1964).

Entre otras actividades, Seguín ha sido impulsor de numerosas publicaciones académicas en el Perú y miembro editorial de revistas internacionales. En 1950 funda la revista Estudios Psicosomáticos, en 1964 crea la Revista de Ciencias Psicológicas y Neurológicas que se publicó hasta 1967, y en 1959 funda y dirige la revista Anales del Servicio de Psiquiatría del Hospital Obrero. Pero además de ello, ha conformado el comité editorial de prestigiosas revistas peruanas e internacionales como la Revista Médica del Hospital Obrero, la Revista de Neuro-psiquiatría, la revista Anales de Salud Mental del Instituto de Salud Mental de Honorio Delgado-Hideyo Noguchi, la Revista Latinoamericana de Psiquiatría, la Revista de Medicina Psicosomática de Buenos Aires, el Journal of Nervious and Mental Disease, el Journal of Ortopsychiatry, el Journal of Psychosomatic Medicine, Acta Psychotherapeutic y el International Journal of Social Psychiatry (Valdivia, 1991). 


\section{Otras facetas de Carlos Alberto Seguín}

Entre sus múltiples facetas, Seguín fue un hombre de familia muy comprometido con los suyos. Tuvo dos hijos, Alberto Gonzalo y María Cristina, pero lamentablemente, su hijo mayor falleció el 4 de enero de 1971 a causa de una gripe mal curada que derivó en una cardiomiopatía viral que ocasionó dos embolias pulmonares, costándole la vida cuando tenía solo 28 años y cuando estaba por sustentar su tesis (Silva, 1979).

Existe un libro dedicado a su hijo, titulado Tú y la Medicina, en el que Seguín brinda diversas orientaciones sobre el rol integral del médico, cuando Alberto Gonzalo decide estudiar la carrera de Medicina (Seguín, 1957). Este libro fue prologado por Florencio Escardó, quien en 1954 publicó una obra de similar contenido con el título El alma del médico (Escardó, 1954). Es además, un texto muy emotivo y está escrito en forma de un diálogo con su hijo a quien se dirige en segunda persona, tocando temas sobre la vocación del médico, la lectura de datos científicos y de literatura, la necesidad de cultivar las artes y la filosofía, la concepción del hombre, la especialización profesional, la vida y la muerte, la religión y el amor; señalando que amar

\footnotetext{
...es ser en plenitud, es salirse uno mismo y sentirse capaz de una fusión con los demás; es renunciar al Yo, es trascender el egoísmo, vivir en comunión y hacerlo activa y gozosamente, con la euforia de una suprema realización y la vivencia de un florecimiento total. Amar es, paradójicamente, cumplir nuestro destino individual, sacrificándolo; realizar nuestro yo más auténtico, diluyéndolo; es ser hombre entre los hombres y para la humanidad. (Seguín, 1957, p. 16)
}

En ese sentido, Seguín, fue uno de los pocos académicos peruanos que escribió sobre el amor con hondura filosófica, histórica, científica y literaria; pues además de Amor y Psicoterapia, publicó en 1976 Amor, sexo y matrimonio, en el que luego de hacer un análisis etimológico, histórico y filosófico del amor, el sexo y el matrimonio, explicando que aunque lo ideal es que estos tres aspectos se acompañen, es posible que haya amor sin sexo y matrimonio, o sexo sin matrimonio y amor, o matrimonio sin amor y sexo; o bien sexo y amor sin matrimonio, matrimonio y amor sin sexo o sexo y matrimonio sin amor (Seguín, 1976). Para ello recurre a explicaciones transculturales y de corte histórico sobre la base de los temas tratados previamente en dicha obra.

Otra faceta de Seguín está íntimamente relacionada con la docencia y su interés por la formación de los médicos y psiquiatras (Seguín, 1957, 1961b, 1963, 1964a; Valdivia, 1964). De hecho, son varios los discípulos suyos que han trascendido en el campo de la medicina, la psiquiatría y el psicoanálisis, como por ejemplo Max Silva, 
quien ha compilado varios de los trabajos de Seguín y ha publicado algunos datos biográficos producto de diversas entrevistas realizadas a su maestro (Silva, 1979, 1994), además de algunos estudios sobre el curanderismo (Silva, 1965), como Mario Chiappe (1969, 1972, 1989), quien también se avocó por la investigación de esta temática, o Saúl Peña, quien entre 1963 y 1966 trabajó con Seguín en el Hospital Obrero y posteriormente se formó en el Instituto de Psiquiatría de la Universidad de Londres y la Sociedad Británica de Psicoanálisis, fundando en 1973 el Royal College of Psychiatrists of England y 1980 la Sociedad Peruana de Psicoanálisis, de la que es presidente honorario desde 1987 (Quezada, 2015). Peña ha sido también presidente de la Federación Psicoanalítica de América Latina y ha publicado varios artículos y libros sobre psicoanálisis, siendo un texto que obtuvo suma difusión: Psicoanálisis de la corrupción. Política y ética en el Perú contemporáneo (Peña, 2003).

Ahora bien, fuera del ámbito académico, Seguín ha desarrollado su obra literaria, pues de sus 374 trabajos publicados, que comprenden libros, artículos y capítulos de libros, 68 se refieren a temas sociológicos, antropológicos, arte y literatura (Valdivia, 1991). En ese sentido, Seguín ha escrito una obra teatral titulada "Encrucijada", que fue galardonada con la Medalla de Oro por la Municipalidad de Lima en 1963, además de los ensayos "Honorio Delgado y el psicoanálisis" y "Lope de Aguirre, el rebelde" (Huarcaya-Victoria, 2016). También ha publicado un libro de cuentos titulado "La calle larga", en el que figura su cuento más conocido "La máquina de escribir" que conjuga la creatividad y el temor al fracaso, que vivencian los hombres de letras; cuyo producto literario, por más insensato que parezca, puede ser interpretado irónicamente de forma vanguardista. También ha escrito un poemario titulado "La noche negra", que contiene "catorce poemas de un amor obscuro".

Algunos de estos textos han sido compilados en el libro Tres facetas (Seguín, 1982), por uno de los discípulos de Seguín, Max Silva; quien además compiló en el libro Seguín periodista (Seguín, 1994) los artículos que Seguín publicaba como columnista en diversos diarios peruanos de los que era asiduo colaborador. Este texto reúne diversos artículos sobre temáticas tales como la labor periodística, que Seguín observó de cerca a través de la obra de su padre; así como el análisis etimológico de diversas palabras o la creación de algunas otras como "veracear" y "ortodecir" para designar una palabra opuesta a mentir, pues “decir la verdad” más que un verbo es una expresión. También contiene artículos sobre el psicoanálisis y la medicina psicosomática; la niñez y la crianza, la vida de pareja y la familia; la agresividad, la violencia y el terrorismo; arte, cine y 
literatura, muy particularmente sobre algunas obras de Mario Vargas Llosa; y sobre su visión humanística del quehacer integral del médico, entre muchos otros temas.

Ya durante su senectud, Seguín recibía constantes visitas de sus discípulos y colegas, con quienes conversaba de diversos temas y compartía sus experiencias personales y profesionales (Perales, 2007). En 1995, pocos días antes de celebrar su cumpleaños 88, la Asociación Psiquiátrica Peruana le iba a rendir un homenaje, pero su vida se apagó repentinamente (Perales, 2008), dejando tras de sí una carrera de logros y enseñanzas inagotables. Luego de su muerte, su hija María Cristina donó los libros de Seguín a la Facultad de Medicina en la UNMSM, antes de volver a Europa. No obstante, años atrás, Seguín ya había donado varios de sus libros a la biblioteca del Hospital Obrero.

\section{Discusión}

Seguín fue un hombre conectado con la psiquiatría de su tiempo, a la que consideraba demasiado cientificista, organicista e individualista. Por ello, desde una aproximación filosófica y antropológica, le brindó una orientación humanista y psicoanalítica sobre la base de la medicina psicosomática y la psiquiatría social, que le llevó a vincularse institucionalmente con diversas asociaciones psiquiátricas y psicológicas, y a participar activamente de congresos internacionales.

Hasta aquí, es posible decir que Seguín fue un hombre que se ha ganado un lugar en la historia de la psiquiatría y la psicología peruanas, que no solo ha trascendido a través de su obra, sino por sus cualidades morales y humanas, porque siempre actuó en congruencia con sus ideales. Con justa razón, Alva (1995) lo describe como médico generoso, viajero impenitente, deportista sempiterno, amante del buen cine, pensador original, conversador ameno, profesional comprometido, cultor de las bellas artes, prolífico escritor y apasionado de la enseñanza. Su obra refleja una activa fuerza que le permitió conquistar diversos campos de la salud mental, primero a través de la medicina psicosomática y la psicoterapia, para luego abocarse a la psiquiatría social y folklórica (Alarcón, 2003); pero siempre con una aproximación psicoanalítica y existencialista que lo inundó de una visión holística del hombre, considerando su mundo interno sin desligarlo de su dimensión cultural.

Aunque Alarcón (1968) dice que Seguín más que un cultor teórico del psicoanálisis fue un promotor de la medicina psicosomática, sus escritos siempre aludieron a las teorías de Freud, que le sirvieron para interpretar las más profundas 
motivaciones humanas. Si bien Seguín se formó como psicoanalista en Nueva York, se ha considerado a Francisco Alarco como el primer psicoanalista peruano (Valdivia, 1991); lo cual es incongruente dado que este renombrado psiquiatra se formó en el Instituto Psicoanalítico de Chicago entre 1954 y 1955, una década después que Seguín.

La trayectoria biográfica, académica y profesional de Seguín ha sido muy variopinta, aunque con una focalización alrededor de su especialidad: la psiquiatría. Ha sido un autor muy prolífico y un entusiasta conferencista internacional. Fue fundador y gestor de varias sociedades de psiquiatría y psicología, así como de revistas académicas. Mantuvo un vivo interés por la formación del médico y particularmente por la enseñanza de la psicología en medicina, que hacia mediados del siglo XX se constituyó en un aspecto central del campo de la formación de los médicos. Se lo reconoce como una figura sobresaliente de la psiquiatría peruana y latinoamericana, que contribuyó a formar nuevos médicos, psiquiatras y psicólogos.

Desde luego, su vida no estuvo exenta de contratiempos y desavenencias, las cuales no le impidieron forjar toda una trayectoria científica y profesional destacada en el Perú y en América Latina. Su producción intelectual combina de manera bastante innovadora cuestiones relacionadas con la filosofía, la antropología, la sociología, el psicoanálisis, el arte, la literatura y el cine. En el contexto de la época que le tocó vivir, contribuyó especialmente al desarrollo de tres áreas de especialización psicológica: medicina psicosomática, psiquiatría social y psicoterapia. Sin dudas, cualquier intento por profundizar en la historia de esas tres áreas en América Latina no pude obviar el nombre de Carlos Alberto Seguín. 


\section{Referencias}

Ackerknecht, E. H. (1962). Breve historia de la psiquiatría. Editorial Universitaria de Buenos Aires.

Alarcón, R. (2003). ¿Psiquiatría folklórica, etno-psiquiatría o psiquiatría cultural? Examen crítico de la perspectiva de Carlos Alberto Seguín. Revista Latinoamericana de Psiquiatría, 8-15. https://psiquiatria.com/bibliopsiquis/volumen.php?wurl=psiquiatria-folkloricaetnopsiquiatria-o-psiquiatria-cultural-examen-critico-de-la-perspectiva-c-aseguin

Alarcón, R. (2011). Carlos Alberto Seguín (1907-1995). En S. Villaseñor, C. Rojas \& J. Garrabé (Eds.). Antología de textos clásicos de la psiquiatría latinoamericana (pp. 357-358). GLADET.

Alarcón, R. (1968). Panorama de la psicología en el Perú. Universidad Nacional Mayor de San Marcos.

Alarcón, R. (1975). El psicólogo y la psicoterapia en el Perú. Revista Interamericana de Psicología, 9(1-2), 47-54. https://journal.sipsych.org/index.php/IJP/article/view/721/618

Alarcón, R. (2000). Historia de la psicología en el Perú. De la Colonia a la Republica. Universidad Ricardo Palma.

Alva, J. (1995). Elogio al maestro. Anales de Salud Mental, 11, 175-182.

Ardila, R. (1971). Acontecimientos importantes en la historia de la psicología latinoamericana. Revista Interamericana de Psicología, 5(1-2), 1-11. https://journal.sipsych.org/index.php/IJP/article/view/603/507

Ardila, R. (1986). La psicología en América Latina. Pasado, presente y futuro. Siglo XXI.

Arias, W. L. (2014). La Sociedad Interamericana de Psicología y los congresos internacionales de la SIP. Revista de Psicología (Universidad Católica San Pablo), $\quad 4, \quad 115-120$. https://revistas.ucsp.edu.pe/index.php/psicologia/article/view/25/26

Arias, W. L. (2015). Honorio Delgado (1892-1969), un repaso histórico sobre su vida y su obra: A propósito de los 100 años del psicoanálisis en el Perú. Boletín de la Academia Paulista de Psicología, 35(89), 286-308.

Arias, W. L. (2016). Contribuciones para una historia local de la psicología en Arequipa. En R. Mardones, Historia local de la psicología. Discusiones teóricas, metodológicas y experiencias de investigación (pp. 354-388). Universidad Santo Tomás.

Ato, M., López-García, J. J., \& Benavente, A. (2013). Un sistema de clasificación de los diseños de investigación en psicología. Anales de Psicología, 29(3), 1038-1059.

Berrios, G. (2011). Hacia una nueva epistemología de la psiquiatría. Editorial Polemos.

Berrios, G. (2018). Historia de los síntomas de los trastornos mentales. La psicopatología descriptiva del siglo XIX. Fondo de Cultura Económica.

Briolotti, A. S. (2017). Pediatría, puericultura y saberes "psi”" en el campo del desarrollo infantil. Una historia de los usos médicos del conocimiento psicológico en el Río de la Plata (1930-1963). (Tesis de Doctorado en Psicología). Universidad Nacional de La Plata, Argentina. https://ri.conicet.gov.ar/handle/11336/82869

Buber, M. (1974/2017). Yo y tú. Herder.

Cabrejos, J. C. (1995). Carlos Alberto Seguín (1907-1995) Semblanza. Anales de la Facultad de Medicina, 56(1), 61-62. https://doi.org/10.15381/anales.v56i1.4937 
Campos-Farfán, C. (2017). El Dr. Raúl González Enríquez y la fundación de la Asociación Psiquiátrica de América Latina. Gaceta Médica de México, 153(3), 406-414.

Caravedo, B., Rotondo, H., \& Mariátegui, J. (Eds.). (1963). Estudios de psiquiatría social en el Perú. Lima: Ediciones del Sol.

Caravedo, B. Rotondo, H., Seguín, C. A., Perales, A., \& Blanco, E. (1971). Comunidad terapéutica en el Perú. Ministerio de Salud Pública - Universidad Nacional Mayor de San Marcos.

Caravedo, B., Seguín, C. A., \& Rotondo, H. (1963). Informe del Perú al Segundo Seminario Latinoamericano de Salud Mental. Boletín de la División de Higiene Mental, 1-78.

Chiappe, M. (1969). Psiquiatría folklórica peruana. Anales del Servicio de Psiquiatría, 11, 1-2.

Chiappe, M. (1972). Los curanderos, los médicos y la enfermedad. Revista del Hospital Obrero, 21, 3-5.

Chiappe, M. (1989). Psiquiatría folklórica peruana: El curanderismo en la costa norte del Perú. Anales de Salud Mental, 5, 190-225. http://repebis.upch.edu.pe/articulos/ansm/v9n1_2/a15.pdf

Contreras, C., \& Cueto, M. (2018). Historia del Perú contemporáneo. 5ta. Edición. Instituto de Estudios Peruanos - Pontificia Universidad Católica del Perú Universidad del Pacífico.

Dagfal, A. (2015). El pasaje de la higiene mental a la salud mental en la Argentina, 19201960. El caso de Enrique Pichon-Rivière. Trashumante, 5, 10-36.

Escardó, F. (1946). La enseñanza de la pediatría. Archivos de Pediatría del Uruguay, 17(9), 505-583.

Escardó, F. (1949). Psicomatismo y medicina infantil. Archivos Argentinos de Pediatría, $32(5)$,

398-405. https://www.sap.org.ar/docs/publicaciones/archivosarg/1949/1949_32_5.pdf

Escardó, F. (1954). El alma del médico. Assandri.

Escardó, F. (1962). Anatomía de la familia. El Ateneo.

Ferreira, A. O. (2010). Movimentos progressivos-regressivos da reforma psiquiátrica antimanicomial no Brasil: Uma Análise da saúde mental na perspectiva da psicologia social crítica. Salud \& Sociedad, 1(3), 165-177. http://dx.doi.org/10.22199/S07187475.2010.0003.00002

Flanders Dunbar, H. (1965). Medicina psicosomática. En Medicina psicosomática y psicoanálisis de hoy (pp. 9-39). Paidós.

Foucault, M. (1987). Enfermedad mental y personalidad. Paidós.

Foucault, M. (1963/2013). El nacimiento de la clínica. Siglo XXI.

Frankl, V. (1987/1997). Teoría y terapia de las neurosis. Iniciación a la logoterapia y el análisis existencial. Herder.

Gallegos, M. (2012a). Historia de la psicología interamericana: Sociedad Interamericana de Psicología (1951). Psychologia Latina, 3(1), 23-36. http://dx.doi.org/10.5209/rev_PSLA.2012.v3.n1.38739

Gallegos, M. (2012b). El Primer Congreso Interamericano de Psicología (1953): su acontecer histórico. Revista Interamericana de Psicología, 46(1), 21-34. https://www.redalyc.org/pdf/284/28424858003.pdf

Gallegos, M., Falcone, R., Franco, I., \& Cervigni, M. (2019). Honorio Delgado en el campo psicológico de América Latina. Revista Peruana de Historia de la Psicología, 5, 25-38. 
Gallegos, M., \& Franco, I. (2020). El psicoanálisis en América Latina: Homenajes a Sigmund Freud. Revista de Psicología, 29(2), 1-8. http://dx.doi.org/10.5354/0719-0581.2020.58169

Gutiérrez Noriega, C. (1936). El temperamento ciclotímico y el tipo pícnico entre los aborígenes peruanos. Actualidad Médica Peruana, 1, 571-578.

Gutiérrez Noriega, C. (1937). Diseño de un estudio psicológico y antropológico de la raza amerinindia. Actualidad Médica Peruana, 3, 159-195.

Gutiérrez Noriega, C. (1939). El pensamiento mágico en las pinturas del antiguo Perú. Revista de Neuropsiquiatría, 2, 426-462.

Heidegger, M. (1987/2003). Introducción a la metafísica. Gedisa.

Hereford, C. F., \& Natalicio, L. (Comps.) (1967). Aportaciones de la psicología ala investigación transcultural. Memorias del Décimo Congreso de la Sociedad Interamericana de Psicología. Trillas.

Huarcaya-Victoria, J. (2016). Carlos Alberto Seguín: A 75 años de su labor asistencial en el Hospital Nacional Guillermo Almenara Irigoyen. Anales de la Facultad de Medicina, 77(4), 403-408. http://dx.doi.org/10.15381/anales.v77i4.12659

Klappenbach, H. (2014). Acerca de la metodología de investigación en la historia de la psicología. Psykhe, 23(1), 1-12. https://doi.org/10.7764/psykhe.23.1.584

León, R., \& Zambrano, A. (1992). Honorio Delgado: Un pionero de la Psicología en América Latina. Revista Latinoamericana de Psicología, 24(3), 401-423. https://www.redalyc.org/pdf/805/80524312.pdf

León, R. (1993). Contribuciones para una historia de la psicología en el Perú. CONCYTEC.

Mariátegui, J. (1981). Hermilio Valdizán. El proyecto de una psiquiatría peruana. Minerva.

Mariátegui, J. (1985). La psiquiatría peruana y sus figuras representativas. Minerva.

Mariátegui, J. (1996). Carlos Alberto Seguín: Figura representativa de la psiquiatría peruana. Anales de Salud Mental, 12, 247-249. http://repebis.upch.edu.pe/articulos/ansm/v12n1_2/a15.pdf

Miro Quesada, C. (1957). Historia del periodismo peruano. Librería Internacional del Perú, S. A.

Orbegoso, A. (2016). Psicología peruana. Los prejuicios detrás de la ciencia. Trujillo: Universidad César Vallejo.

Paredes, N. (2019). Historia de la psicología de Arequipa. Antares Impresores.

Peña, S. (2003). Psicoanálisis de la corrupción. Peisa.

Peña, S. (2010). Seguín y la psicoterapia. Aspectos de su vida y obra. Revista de Neuropsiquiatría, 73(4), 164-169. https://doi.org/10.20453/rnp.v73i4.1702

Perales, A. (2007). Carlos Alberto Seguín: Paradigma Sanmarquino. Boletín Médico, 43, $15-16$.

Perales, A. (2008). Carlos Alberto Seguín: Paradigma docente de la Facultad de Medicina de San Fernando, a 100 años de su nacimiento. Anales de la Facultad de Medicina, 69(1), 59-61. https://doi.org/10.15381/anales.v69i1.1188

Perales, A. (2013). Acerca del estudio de la psicopatología general y transcultural [Carta a editor]. Revista Peruana de Medicina Experimental y Salud Pública, 30(1), 155156. http://www.scielo.org.pe/pdf/rins/v30n1/a37v30n1.pdf

Plotkin, M. B., \& Ruperthuz, M. (2017). Estimado Doctor Freud. Una historia cultural del psicoanálisis en América Latina. Edhasa.

Porturas, F. (1999). Origen de la Universidad Peruana Cayetano Heredia. Revista Médica Herediana, 10(4), 151-166. https://doi.org/10.20453/rmh.v10i4.618 
Quezada, J. (2015). Dr. Saúl Peña Kolenkautsky, médico psiquiatra y psicoanalista. $\begin{array}{llll}\text { Avances en } & \text { Psicología, } & \text { 23(2), } & \text { 241-243. }\end{array}$ https://doi.org/10.33539/avpsicol.2015.v23n2.186

Rogers, C. R. (1997). Psicoterapia centrada en el cliente. Paidós.

Romero, J. (2007). Carlos Alberto Seguín. A 100 años del natalicio de un peruano ilustre. Liberabit, 13. http://www.scielo.org.pe/pdf/liber/v13n13/a12v13n13.pdf

Rotondo, H. (1949). La preparación psicosomática del médico práctico. Revista de Neuro-psiquiatría, 12, 301-311.

Rotondo, H. (1959). Morbilidad psiquiátrica en un área urbana en estado de desorganización. Revista Psiquiátrica Peruana, 2, 132-137.

Rotondo, H. (1967). Subdesarrollo, pobreza y obstáculos a los Programas de Salud Mental en la comunidad. Revistas de Ciencias Psicológicas y Neurológicas, 4, 240-252.

Rotondo, H., Mariátegui, J., Bambarén, C., García, C., \& Aliaga, P. (1962). Personalidad básica, migración y salud mental. Boletín de la Oficina Sanitaria Panamericana, 52, 140-142. https://iris.paho.org/handle/10665.2/15155

Sal y Rosas, F. (1965). Procedimientos médicos primitivos de los indígenas peruanos. Revista de la Sanidad de la Policía, 25, 170-176.

Sal y Rosas, F. (1967). Prácticas mágicas, diagnósticas y pronóstico en los indígenas peruanos. Revista de Neuropsiquiatría, 30, 165-190.

Sartre, J.-P. (1943/2008). El ser y la nada. Posada.

Seguín, C. A. (s/f). La anamnesis psicosomática. s/e.

Seguín, C. A. (1938). ¿Qué es el inconsciente? Hijo mío, 144-145, 176.

Seguín, C. A. (1940a). Breve historia del psicoanálisis. Viva Cien Años, 762-764.

Seguín, C. A. (1940b). Freud un gran explorador del alma. Su vida, su obra, el psicoanálisis. Editorial Orientación Integral.

Seguín, C. A. (1946a). The concept of disease. Psychosomatic Medicine, 8, 252-257. http://dx.doi.org/10.1097/00006842-194607000-00004

Seguín, C. A. (1946b). La doctrina psicosomática en el plan de estudios médicos. Revista Médica del Rosario, 36(10), 645-648.

Seguín, C. A. (1947). Introducción a la medicina psicosomática. Scheuch.

Seguín, C. A. (1949). A note on the concept of cure. Psychosomatic Medicine, 11, 305306. http://dx.doi.org/10.1097/00006842-194909000-00009

Seguín, C. A. (1952). Un síndrome común en la práctica hospitalaria. Revista del Hospital Obrero, 1, 1-3.

Seguín, C. A. (1955). Bases de la psicoterapia. En Actas del Primer Congreso Interamericano de Psicología 10-20 de Diciembre de 1953 (pp. 454-464). Sociedad Interamericana de Psicología.

Seguín, C. A. (1957). Tú y la medicina. Ediciones Asandri.

Seguín, C. A. (1956). Migration and psychosomathic disadaptation. Psychosomatic Medicine, 18(5), 404-409. http://dx.doi.org/10.1097/00006842-19560900000004

Seguín, C. A. (1960). Existencialismo y psiquiatría. Paidós.

Seguín, C. A. (1961a). Erasistrato, Antioco y la medicina psicosomática. Anales del Servicio de Psiquiatría (Hospital Obrero), 1, 33-40.

Seguín, C. A. (1961b). Enseñanza de la psiquiatría en Latinoamérica. Anales del Servicio de Psiquiatría (Hospital Obrero), 3(5), 3-12.

Seguín, C. A. (1962). Psiquiatría y sociedad. Estudios sobre la realidad peruana. Universidad Nacional Mayor de San Marcos.

Seguín, C. A. (1963). Amor y psicoterapia. Paidós. 
Seguín, C. A. (1964a). La preparación psicológica del estudiante de medicina. Universidad Nacional Mayor de San Marcos.

Seguín, C. A. (1964b). Sobre una definición de la psicología médica. Revista de Ciencias Psicológicas y Neurológicas, 1(2), 164-177.

Seguín, C. A., \& Ríos, R. (Eds.) (1964). Anales del III Congreso Latinoamericano de Psiquiatría 25 al 31 de Octubre. Asociación Psiquiátrica de América Latina.

Seguín, C. A. (1965). El abrirse, el amar y el permitirse en psicoterapia. Seminario de Medicina, 127-129.

Seguín, C. A. (1969). Psiquiatría folklórica. En O. Valdivia, \& A. Péndola (Eds.). Psiquiatría peruana. Primer Congreso Nacional de Psiquiatría 26-31 de Oct (pp. 154-159). Asociación Psiquiátrica Peruana.

Seguín, C. A. (1970). Teoría y práctica de la psicoterapia en el Perú. Anales del Servicio de Psiquiatría (Hospital Obrero), 12(4), 126-156.

Seguín, C. A. (1971). Folklore Psychiatry. En S. Arieti (Ed.) The World Biennal o Psychiatry and Psychotherapy. Basic Books.

Seguín, C. A. (1972). Ethno-psychiatry and folklore psychiatry. Interamerican Journal $\begin{array}{lll}\text { of } & \text { Psychology, 6(1-2), } & \text { 75-80. }\end{array}$

https://doi.org/10.30849/rip/ijp.v6i1\%20\&\%202.633

Seguín, C. A. (1976). Amor, sexo y matrimonio. Ediciones Esrmar.

Seguín, C. A. (1979). Psiquiatría folklórica. Ediciones Esrmar.

Seguín, C. A. (1982). Tres facetas. s/e.

Seguín, C. A. (1988). Medicinas tradicionales y medicina folklórica. Banco Central de Reserva del Perú.

Seguín, C. A. (1990a). El quinto oído. Ediciones Libro Amigo.

Seguín, C. A. (1990b). El síndrome psicosomático de desadaptación. Anales de Salud Mental, 6, 135-143. http://repebis.upch.edu.pe/articulos/ansm/v6n1_2/a9.pdf

Seguín, C. A. (1994). Seguín Periodista. Ediciones Libro Amigo.

Seguín, C. A. (1953/2007). Bases de la psicoterapia. Colegio de Psicólogos del Perú.

Seguín, C. A., \& Buckley, I. de (1971). Sobre la comunidad terapéutica. En B. Caravedo, H. Rotondo, C. A. Seguín, A. A. Perales \& E. Blanco (Eds.). Comunidad Terapéutica en el Perú (pp. 107-118). Ministerio de Salud Pública - Universidad Nacional Mayor de San Marcos.

Selye, H. (1960). La tensión en la vida (el estrés). Compañía General Fabril Editora.

Silva, M. (1965). El curanderismo en Lima. Revista de Ciencias Psicológicas y Neurológicas, 2, 16-51.

Silva, M. (1979). Conversaciones con Seguín. Mosca Azul.

Silva, M. (1994). Carlos Alberto Seguín. Otros perfiles, otros frentes. Banco Central de Reserva del Perú.

Szasz, T. S. (1970/2006). La fabricación de la locura. Kairós.

Valdivia, O., \& Péndola, A. (1969). Psiquiatría peruana. Primer Congreso Nacional de Psiquiatría Peruana 26-31 octubre, Lima: Asociación Psiquiátrica Peruana.

Valdivia, O. (1964). Historia de la psiquiatría peruana. s/e.

Valdivia, O. (1991). Panorama a de la psiquiatría en el Perú. Universidad Nacional Mayor de San Marcos.

Valdizán, H. (1913). El cocainismo y la raza indígena. La Crónica Médica, 30(594), 168275.

Valdizán, H. (1918a). La chicha, bebida de los antiguos peruanos. Revista de Psiquiatría y Disciplinas Conexas, 1(1), 62-77.

Valdizán, H. (1918b). La psicoterapia extrapsiquiátrica. Anales de la Facultad de Medicina, 1, 250-271. https://doi.org/10.15381/anales.v1i0.10699 
Valdizán, H. (1925). Alienación mental en la raza india. Anales de la Facultad de Medicina, 7, 145-163. https://doi.org/10.15381/anales.v10i0.10229

Valdizán, H. (1990). Paleopsiquiatría del Perú antiguo. Universidad Peruana Cayetano Heredia

Received: 2021-07-22

Accepted: 2021-12-17 